\title{
SURROGATE MODELING \& OPTIMIZATION OF A NONLINEAR BATCH REACTOR BY POLYNOMIAL CHAOS EXPANSION
}

\author{
Nishat Tasnim, Mysha Momtaz and Nahid Sanzida* \\ Department of Chemical Engineering, Bangladesh University of Engineering and Technology (BUET) \\ Dhaka 1000, Bangladesh
}

\begin{abstract}
The paper presents a computationally efficient approach to develop a nonlinear data driven input/output model between the finite-time control trajectories and the quality index at the end of the batch. Polynomial chaos expansion (PCE) was applied to produce the approximate representation of the full process model of a nonlinear batch reactor with the reaction scheme $\mathrm{A} \stackrel{k_{1}}{\rightarrow} \mathrm{B} \stackrel{k_{2}}{\rightarrow} \mathrm{C}$. A surrogate model was developed to estimate the dependence of intermediate product (B) concentration at the end of the batch on the temperature trajectory applied during the reaction. The surrogate model was then validated for its performance. Later, the surrogate model was used to determine the optimal temperature profile needed to maximize the concentration of intermediate product at the end of the batch. The validation and optimization results prove that the experimental data based PCE can provide a very good approximation of the desired outputs, providing a generally applicable approach for rapid design, control and optimization of batch reactor systems.
\end{abstract}

Keywords: Batch Reactor, Surrogate Model, Polynomial Chaos Expansion, Optimization.

\section{Introduction}

The batch processes are responsible for the operation of $40-60 \%$ of chemical process industries [1] including pharmaceuticals, polymers, food products, biotechnology, and electronic chemicals [2]. However, due to the absence of steady state operating point, repetitive nature, non-linear behavior, constrained operation with few specific measurements, presence of disturbances [3], it has become a challenging issue for engineers to understand, model development, and control of batch chemical reactor [4]. Considering the above fact, an urgency has been increasing on control of complex distributed parameter chemical systems due to the boost of computing power, remarkable advancement in sensor and actuator, and the development of modern optimization and model reduction algorithms [5].

There is a common practice in the batch process industries to overlook the difficulties associated with it and design batch processes using conventional methods approximating lumped models. From singleinput-single-output proportional-integral-derivative (SISO PID) controllers to plant wide Model Predictive Control (MPC) systems, there are multifaceted applications of feedback control systems, which implicitly or explicitly presume that process dynamics are either inherently linear or almost linear owing to process operation close to a steady state [6]. But this model is often insufficient to describe the process - dynamics satisfactorily due to the inherent nonlinearity of batch process. This insufficiency leads to the increase interest in Nonlinear Model Predictive Control (NMPC) which refers to MPC schemes that are based on nonlinear models and /or consider nonquadratic cost-functional and general nonlinear constraints on the states and inputs [7]. Though nonlinear control usually poses substantially higher data, design, implementation, and maintenance demands than linear control [8], it is still a predicament whether nonlinear control strategy will be advantageous over linear control alternatives due to the time and effort required to develop a non-linear model [9]. As both of linear and nonlinear MPC are first principle model based approaches, their development is complicated, time-consuming and also expensive.

In this scenario, the monitoring and robust optimal control of batch processes diverts towards an alternate simple operating data driven and computationally efficient control strategies. Surrogate modeling has been used in the process design and optimization purpose which is created to estimate the computationally expensive simulation codes. They can be used to replace the exact analysis of evolutionary search and can also provide an understanding of the functional relationship between the input and output [10]. To develop a nonlinear data based control strategies for batch chemical reactors polynomial chaos expansion (PCE) based surrogate 
modeling is used for system identification and optimization techniques. After being introduced by Norbert Wiener in 1938 for turbulence modeling [11], PCE has been used in diverse application over the decades which offers an efficient high-order precise way of including non-linear effects in stochastic analysis.

In case of optimal control of processes operating close to safety and performance constraints, uncertainty analysis of static and dynamic system models is essential since almost all mathematical models inherently hold uncertainty in form of perturbations and disturbances [12]. The development of model based computation without considering the uncertainty in the observed data, model parameters and implemented inputs may cause failure in optimal control [13]. Uncertainty is usually quantified by characterizing the effects of uncertainty on theoretical model of actual system which may be parametric model perturbations, lack of physical fidelity of models and uncertainty circumstances in system operation [13]. Monte Carlo evaluations are quiet expensive due to requiring large number of samples, are not sufficient for large and complex models and the single deterministic simulations also sometimes demand parallel high performance computing [14]. Polynomial chaos expansion as a functional approximation of mathematical model, has proved better performance in diverse systems including finite elements and computational fluid dynamics [15]. Defining a system as a polynomial assuming that a finite sum of polynomials can accurately approximate the function of interest is a general trend nowadays where orthogonal polynomials are often used [16] though different orthogonal functions are optimal for different parameter probability density functions. PCE is expressed as an expansion of multidimensional Hermite polynomial functions of the uncertain parameters as it contains orthogonal basis with respect to Gaussian probability measure. A non-linear system can be replaced with surrogate model by PCE that depicts the input-to-state and input-to-output behavior within a fixed range. As PCE is convergent in the mean-square sense [17], least-squares minimization can be utilized to calculate the coefficients through the consideration of sample input-output or experiment.

PCE has been applied in electrical measurement, electric circuit models, chemical processes, biotechnological processes, reaction engineering, transport phenomena, batteries, robot manipulators, helicopters, mechanical systems etc. Stability analysis, parameter estimation, optimal trajectory computation, robust optimal linear quadratic regular design, stochastic optimal control, filtering, observing design, design of stochastic model predictive controllers and many other applications are notable [13].
In this paper, polynomial chaos expansion (PCE) is used to develop a nonlinear surrogate model of a batch chemical reaction process. Performance analysis was also performed on the surrogate model to evaluate its accuracy. Later this model was used to optimize the temperature profile required to obtain a desired concentration of the intermediate product at the end of the batch.

\section{Polynomial Chaos Expansion (PCE)}

If the input temperature trajectory described in terms of standard normal random variables, the polynomial chaos expansion (PCE) can describe the model output $\Psi$ as an expansion of multidimensional Hermite polynomial functions of the input parameters $\theta$ [18]. Using the Hermite bases in the PCE, the output can be expressed in terms of the standard random normal variables $\left\{\theta_{i}\right\}$ using an expansion of order $d$ as shown in Equation 1,

$$
\begin{aligned}
& \psi^{(d)}=\underbrace{a_{o}^{(d)} \Gamma_{o}}_{\text {constant }}+ \\
& \underbrace{\sum_{i_{1}=1}^{n_{\theta}} a_{i_{1}}^{(d)} \Gamma_{1}\left(\theta_{i_{1}}\right)}_{\text {first-order terns }}+\underbrace{\sum_{i_{1}=1}^{n_{\theta}} \sum_{i_{2}=1}^{i_{1}} a_{i_{1} i_{2}}^{(d)} \Gamma_{2}\left(\theta_{i_{1}}, \theta_{i_{2}}\right)}_{\text {second-order terns }}+ \\
& \underbrace{\sum_{i_{1}=1}^{n_{\theta}} \sum_{i_{2}=1 i_{i_{3}}=1}^{i_{1}} \sum_{i_{2}}^{i_{2}} a_{i_{1} i_{2} i_{3}}^{(d)} \Gamma_{3}\left(\theta_{i_{1}}, \theta_{i_{2}}, \theta_{i_{3}}\right)}_{\text {third-order terns }}+\ldots
\end{aligned}
$$

where, $n_{\theta}$ is the number of parameters, $a_{i_{1}}^{(d)}, a_{i i_{2}}^{(d)}$, and $a_{i_{i} i_{i} i_{3}}^{(d)}$ are the deterministic coefficients to be estimated, $\Gamma_{1}, \Gamma_{2}$, and $\Gamma_{3}$ are the successive polynomial chaoses of their arguments, the subscripts denote the order of the expansion which is convergent in the mean square. A combined alternative expression for PCE with degree $m=i_{1}, i_{2}, \ldots, i_{n_{\theta}}, \Gamma_{m}\left(\theta_{i_{1}}, \ldots, \theta_{m}\right)$ is,

$\Gamma_{m}\left(\theta_{i_{1}}, \ldots, \theta_{m}\right)=(-1)^{m} e^{(1 / 2) \theta^{\theta_{\theta}} \theta} \frac{\partial^{m} e^{-(1 / 2) \theta^{T_{\theta}}}}{\partial \theta_{1} \ldots \partial \theta_{m}}$

The polynomial chaos terms are random variables, since they are functions of the random variables, and terms of different order are orthogonal to each other (with respect to an inner product defined in Gaussian measures as the expected value of the product of the two random variable, i.e., $\varepsilon\left[\Gamma_{i} \Gamma_{j}\right]=0$ for $\Gamma_{i} \neq \Gamma_{j}$. The number of coefficients $(N)$ in the PCE depends on the number of uncertain parameters $\left(n_{\theta}\right)$ and the order of expansion $(m)$.

The general formula for the determination of number of coefficients is expressed by Equation 3 .

$N=1+\frac{n_{\theta} !}{\left(n_{\theta}-1\right) ! !}+\frac{\left(n_{\theta}+1\right) !}{\left(n_{\theta}-1\right) ! 2 !}+\ldots+\frac{\left(n_{\theta}+m-1\right)}{\left(n_{\theta}-1\right) ! m !}$ 
Since, the PCE is convergent in the mean-square sense it is beneficial to calculate the coefficients using least-square minimization (LSM) considering sample input/output pairs from the model. The optimization is performed until the best fit is achieved between the surrogate PCE and the nonlinear model (or experimental data).

\section{PCE Based Surrogate Modeling and Validation of a Batch Reactor System}

In this study a nonlinear batch reactor has been taken into consideration. Here temperature is the control variable [19]. The objective of the control problem is to maximize the intermediate product (B) after a certain reaction time. The sequence of the reactions is presented in Equation 4,

$\mathrm{A} \stackrel{k_{1}}{\rightarrow} \mathrm{B} \stackrel{k_{2}}{\rightarrow} \mathrm{C}$

where, $k_{1}, k_{2}$ are the rate constants for the production of $\mathrm{B}$ and $\mathrm{C}$ respectively. The rate equations describing the batch process are as shown in Equation 5 and Equation 6,

$\frac{\mathrm{d} c_{1}}{\mathrm{~d} t}=k_{1} \exp \left(-E_{1} / v T_{r e f}\right) c_{1}^{2}$

$\frac{\mathrm{d} c_{2}}{\mathrm{~d} t}=k_{1} \exp \left(-E_{1} / v T_{\text {ref }}\right) c_{1}^{2}-k_{2} \exp \left(-E_{2} / v T_{\text {ref }}\right) c_{2}$

where, $c_{1}$ and $c_{2}$ are the dimensionless concentration of A and B respectively; $v=T / T_{\text {ref }}$ is the dimensionless temperature of the batch reactor and $T_{\text {ref }}$ is the reference temperature. The final time $t_{f}$ is fixed to be 1 hour; the values of the parameters $k_{1}, k_{2}, E_{1}$, and $E_{2}$, are given in Table 1 .

Table 1

Parameter Values for Batch Reactor [19]

\begin{tabular}{cc}
\hline Parameter & Values \\
$k_{1}$ & $4 \times 10^{3}$ \\
$k_{2}$ & $6.2 \times 10^{3}$ \\
$E_{1}$ & $2.5 \times 10^{3}$ \\
$E_{2}$ & $5.0 \times 10^{3}$ \\
$T_{r e f}$ & $348 \mathrm{~K}$ \\
\hline
\end{tabular}

The initial conditions are kept at $c_{1}(0)=1, c_{2}(0)=1$ and the batch reactor temperature is constrained between $298 \mathrm{~K}<T<398 \mathrm{~K}$.

Based on the above information, a rigorous simulation program was developed in MATLAB $®$ and was treated as the real process. Initially, the batch length is divided into $\mathrm{N}=10$ equal stages. Twenty batches of process operations under different temperature profiles were simulated using the MATLAB ${ }^{\circledR}$ model. These twenty sets of historical data were used as training data. The training data was generated to ensure the diverse behaviour of the state for capturing the system's response precisely. The inputs were the temperature trajectories discretized in 9 points (hence 10 input parameters, $\theta_{i}$ ) over the batch duration of 1 hour. The output was the corresponding concentration $c_{2}$ of intermediate product, $\mathrm{B}$ at the end of the batch.

The batch rector system was then re-identified by a $2^{\text {nd }}$ order nonlinear Polynomial Chaos Expansion (PCE) based surrogate model using these training data. As shown before, for second order PCE with 10 parameters, the numbers of coefficients are 66. The coefficients of PCE were calculated by least square (LSM) method where a set of randomly chosen initial coefficients were applied and the simulation was carried out through trial and error basis using the former coefficients for the later trial until the prediction accuracy achieved no further progression. Figure 3 (a) represents the concentration values obtained from both the first principle model and PCE based model with respect to number of batches. It shows that the values obtained from PCE have a highest diversion by $0.65 \%$ from the actual model values. The straight line in the last graph (Figure 3 (b)) stands for the actual model values and the values obtained from PCE is noticed to be scattered around the straight line clearly.
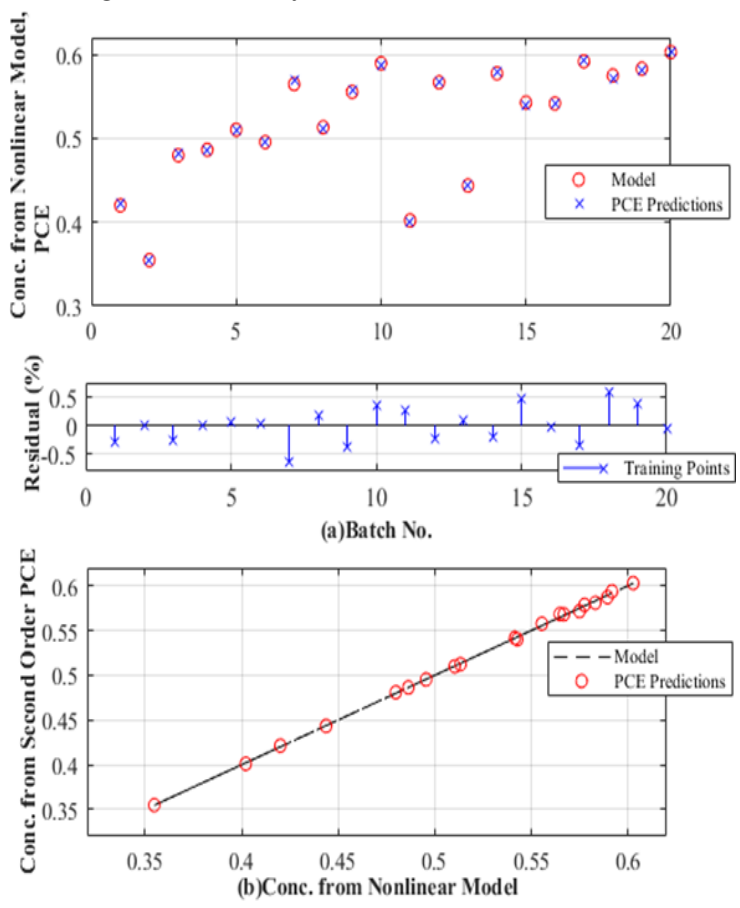

Fig. 3: Prediction of the 2nd order PCE (a) for 20 training batches (b) comparison of PCE prediction with nonlinear model

The surrogate model was validated then using different input temperature profile other than the training data set. Two types of input temperature profiles were used for this validation. Some input temperature profiles yield output concentration, $c_{2}$ within the range of training data set. The other set which was outside the range used for checking the 
extrapolation ability. The results obtained from second order PCE validation is shown in Figure 4. It took about 5 minutes to complete the simulation in MATLAB R2020a of second order using the personal computer with Microsoft Windows 10 operating system and Intel Core i5, $8^{\text {th }}$ Gen processor with $4 \mathrm{~GB}$ RAM. The sum squared error was calculated as $\mathrm{SSE}=\sum$ (actual concentration obtained from nonlinear model-calculated concentration from surrogate model $)^{2}$. The results of model identification and validation have been listed in the Table 2 .

\section{Table 2}

Actual and Calculated Concentration SSE Values by Second Order PCE

\begin{tabular}{ll}
\hline & SSE \\
\hline Surrogate model identification & $7.64 \times 10^{-03}$ \\
Validation within the limit of input trajectory & $1.15 \times 10^{-05}$ \\
Validation outside the limit of input trajectory & $9.18 \times 10^{-04}$ \\
\hline
\end{tabular}
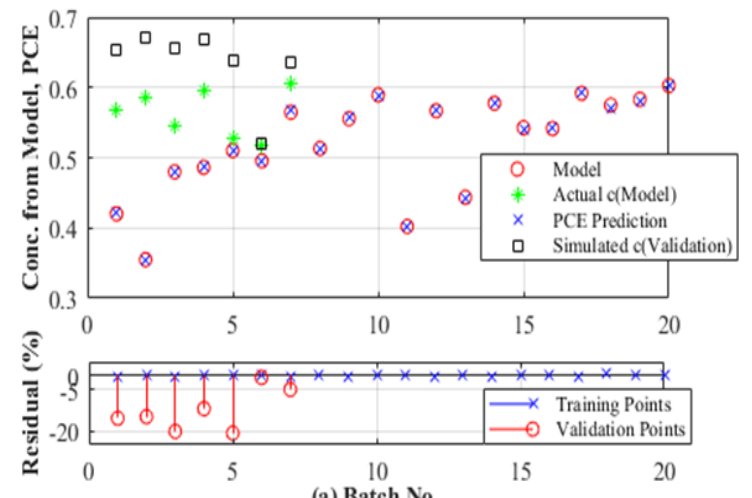

(a) Batch No.

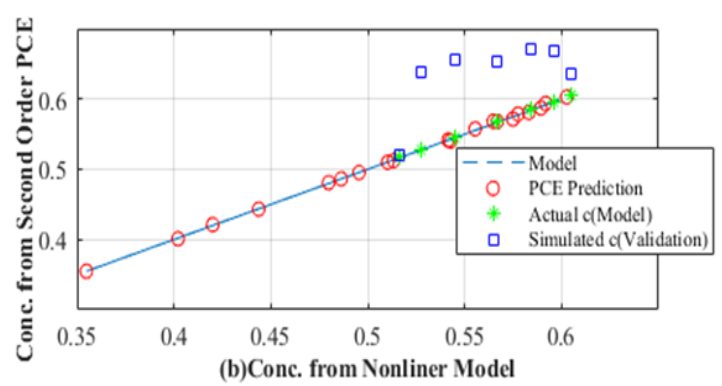

Fig. 4: Prediction of second order PCE (a) for 20 training batches and 7 validation batches (b) comparison of PCE prediction with nonlinear model

\subsection{Optimization of PCE Based Surrogate Model}

The desired concentration, $c_{2}$ of the intermediate product B was at first determined from the optimization of the first principal non-linear model of the batch reactor system. This desired concentration was set as output into the developed PCE based surrogate model to justify its performance and the corresponding optimal temperature profile at the end of the batch was determined.
First Principal Model Based Open Loop Optimal Control

The simulated first principal model of the batch reactor was used for performance optimization purpose where the optimized time profile of the manipulated variable i.e. reactor jacket temperature as determined. The objective function was to maximize the intermediate product concentration $\left(c_{2}\right)$ leading to the optimal control problem of Equation 7.

$$
\max _{\mathrm{T}(1) \mathrm{T}(2), \ldots . ., \mathrm{T}(\mathrm{N})} c_{2}
$$

The system is subjected to the following constraints,

$T_{\min } \leq T(k) \leq T_{\max }$

$R_{\min } \leq \mathrm{d} T / \mathrm{d} t \leq R_{\max }$, and

$c_{1}(0)=1, c_{2}(0)=1$

where, $T_{\min }, T_{\max }, R_{\min }$ and $R_{\max }$ are the minimum and maximum temperatures and temperature ramp rates, respectively, during the batch. A sequential quadratic programming (SQP) approach was implemented in the MATLAB function fmincon to solve the optimization problem. Figure 5 shows the optimal temperature trajectory and the corresponding intermediate product concentration $\left(c_{2}\right)$ trajectory. The optimal temperature profile is decreasing with time while the corresponding intermediate product concentration reaches the maximum value equal to 0.6093 at the end of the batch.

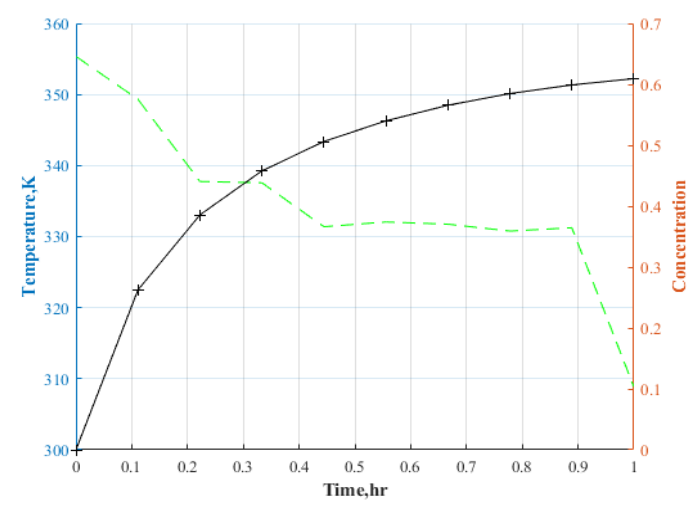

Fig. 5: Optimum temperature profile and corresponding concentration profile for first principle model based optimization

Determination of the Optimal Temperature Profile by Surrogate Model to Produce Maximum Intermediate Product Concentration

The nonlinear surrogate model was then used to determine the optimal temperature profile needed to obtain the desired intermediate product concentration $\left(c_{2}\right)$ of 0.6093 . The objective function is shown in Equation 8. 


$$
\min _{T(1), T(2), \ldots, T(N)}\left(\frac{\text { Desired } c_{2}-c_{2, \mathrm{PCE}}}{\text { Desired } c_{2}} \times 100\right)^{2}
$$

The system was subjected to the following constraints,

$$
\begin{aligned}
& T_{\min } \leq T(\mathrm{k}) \leq \underline{T}_{\max } \quad \text { and } \\
& R_{\min } \leq \mathrm{d} T / \mathrm{d} t \leq R_{\max }
\end{aligned}
$$

Where, $T_{\min }, T_{\max }, R_{\min }$ and $R_{\max }$ were the same as those for the first principal model based optimization. The minimum and maximum temperatures were $298 \mathrm{~K}$ and $398 \mathrm{~K}$ respectively. The temperature ramp rates were, $-1<\mathrm{d} T / \mathrm{d} t \leq 0 \mathrm{~K} / \mathrm{hr}$. These inequality constraints ensure that the optimized temperature profile is implementable. The surrogate model based optimization has been demonstrated by two cases as described below.

Case 1a: Optimization of the Surrogate Model Using a Linear Initial Temperature Profile with Fixed Terminal Conditions on Temperature

In this case, the initial profile was a linear cooling temperature trajectory that produced an intermediate concentration $\left(c_{2}\right)$ of 0.5388 . The cooling temperature was optimized where the lower and upper bound temperature were kept constant at the values $298 \mathrm{~K}$ and $398 \mathrm{~K}$ respectively. During application to the theoretical non-linear model, the optimum temperature calculated by the second order PCE generated an actual concentration of 0.6069 at the end of the batch with the SSE of $5.76 \times 10^{-6}$. The results for temperature and concentration trajectories are shown in Figure 6(a) and 6(b) respectively.

Case 1b: Optimization of the Surrogate Model Using a Nonlinear Temperature Profile with Fixed Terminal Conditions on Temperature

In this case, in an effort to further improve the performance of the surrogate model, the initial profile was changed to a nonlinear cooling temperature profile that produced a final concentration of 0.5429 . The lower and upper bound of temperature were kept constant at the values $298 \mathrm{~K}$ and $398 \mathrm{~K}$ respectively during optimization of the reaction temperature, similar to Case 1a. When applied to the theoretical nonlinear model, the optimum temperature calculated by the second order PCE produced an actual concentration of 0.6069 at the end of the batch with the SSE of $5.76 \times 10^{-6}$. The results for temperature and concentration trajectories are shown in Figure 7(a) and 7(b) respectively.

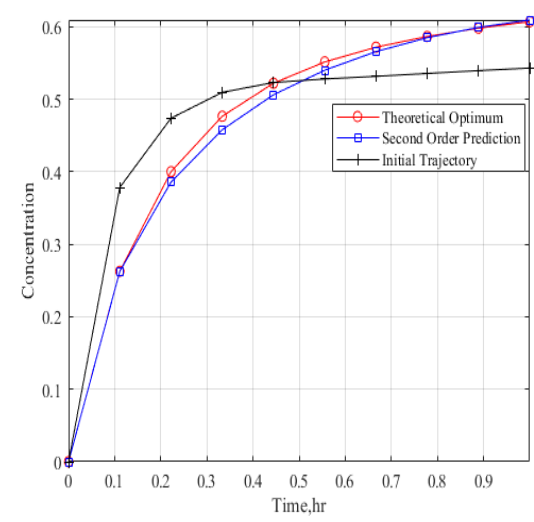

Fig. 6 (a): Optimum temperature profile for cas 1 1a

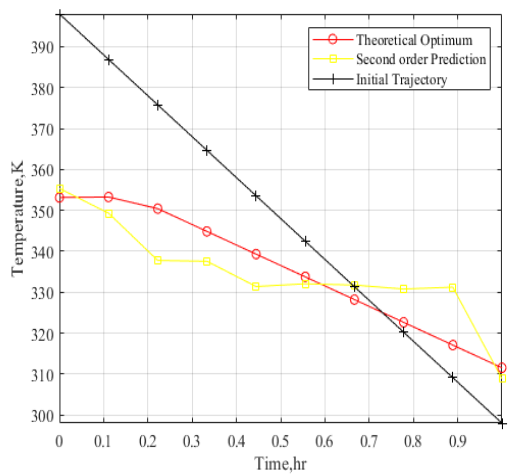

Fig. 6 (b): Optimum concentration profile for case 1a

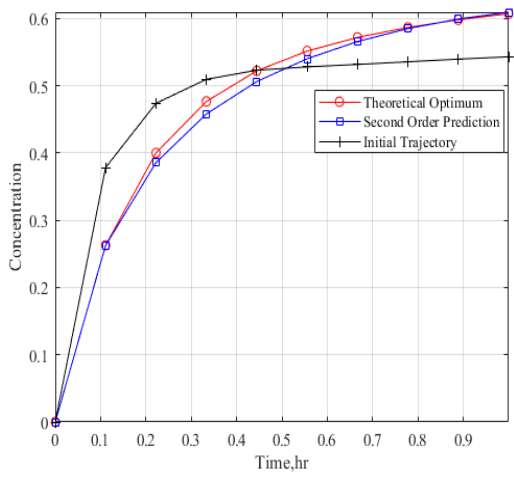

Fig. 7 (a): Optimum temperature profile for case $1 \mathrm{~b}$

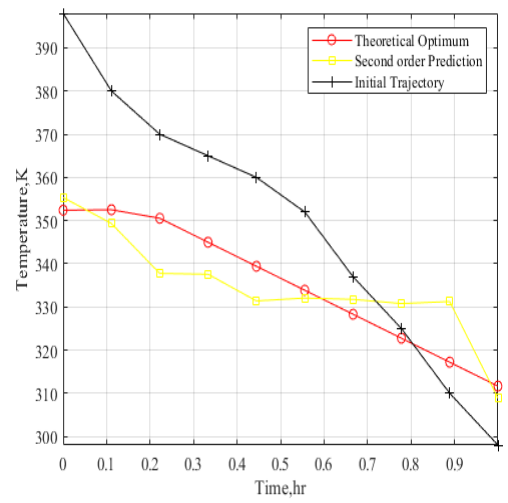

Fig. 7 (b): Optimum concentration profile for case 1 
From the observation of the optimization results, it is notable that as the final concentration of initial trajectory by nonlinear temperature profile $(0.5429)$ is greater than that of linear profile $(0.5388)$, it was expected that the final concentration by second order prediction for nonlinear case would produce a value closer to the theoretical optimum concentration (0.6093) than the linear case. However, the final concentration obtained by the second order prediction in both case $1 \mathrm{a}$ and $1 \mathrm{~b}$ are same (0.6069) and the resulting curves also show little differences. This issue can be solved by increasing the order of polynomial chaos expansion which will also increase the number of coefficients and capture the nature of the actual model more accurately. Usually, an increase upto 4th order is enough for most engineering applications.

\section{Conclusion}

In this study, a polynomial chaos expansion (PCE) based operating data-driven nonlinear surrogate modelling approach has been formulated for a batch chemical reactor system. The performance of the surrogate model was first validated and later optimized to generate the required temperature profile to obtain the desired intermediate product concentration at the end of the batch. The PCE approach used least square minimization (LSM) to calculate the coefficients. The initial assumption of the PCE coefficients played a vital role during all the simulations. So, the overall bottleneck of the PCE based nonlinear surrogate modeling can be identified to be the initial guess of coefficients (e.g. $a_{i_{1}}^{(d)}, a_{i_{1} i_{2}}^{(d)}$, and $\left.a_{i_{1} i_{2} i_{3}}^{(d)}\right)$. The developed surrogate model showed reasonable overfitting during validation and optimization. The residual error was on average $12.69 \%$ during validation (Figure 4). The validation and optimization results prove that the experimental data based PCE can provide a significant approximation of the desired outputs, providing a generally applicable approach for rapid design, control, and optimization of batch chemical reaction systems.

\section{References}

[1] Y. Wang, J. Shi, D. Zhou and F. Gao, "Iterative Learning Fault-tolerant Control for Batch Processes," Industrial \& Engineering Chemistry Research, vol. 45, pp. 9050-9060, 2006.

[2] Z. Nagy and R. Braatz, "Robust Nonlinear Model Predictive Control of Batch Processes," AIChE Journal, vol. 49, pp. 1776-1786, 2003.

[3] D. Bonvin and G. Francois, 2016. [Online]. Available: https://infoscience.epfl.ch/record/232594/files/CR_C ontrolandOptimization_chap11.pdf. [Accessed 18 March 2019].
[4] E. Ekpo, "Dynamic Optimisation and Control of Batch Polymerisation Process," UK, 2006.

[5] R. Roman, Z. Nagy, M. Cristea and P. Agachi, "Dynamic Modelling and Nonlinear Model Predictive Control of a Fluid Catalytic Cracking Unit," Computers and Chemical Engineering, vol. 33, pp. 605-617, 2009.

[6] S. Qin and T. Badgewell, "A Survey of Industrial Model Predictive Control Technology," Control Engineering Practice, vol. 11, pp. 733-764, 2003.

[7] R. Findeisen, L. Imsland, F. Allgöwer and B. Foss, "State and Output Feedback Nonlinear Model Predictive Control: An Overview," European Journal of Control, vol. 9, no. 2-3, p. 190, 2003.

[8] M. Nikolaou and P. Misra, "Linear Control of Nonlinear Processes: Recent Developments and Future Directions," Computers and Chemical Engineering, vol. 27, pp. 1043-1059, 2003.

[9] D. Seborg, "A Perspective on Advanced Strategies for Process Control," Advances in Control, pp. 103-34, 1999.

[10] S. Ong, B. Nair and J. Keane, "Evolutionary Optimization of Computationally Expensive Problems via Surrogate Modeling," AIAA Journal, vol. 41, no. 4, pp. 687-696, 2003.

[11] N. Wiener, "The Homogeneous Chaos," American Journal of Mathematics, vol. 60, pp. 897-936, 1938.

[12] D. Rippin, "Simulation of Single and Multiproduct Batch Chemical Plants for Optimal Design and Operation," Reliability Engineering \& System Safety, vol. 104, pp. 15-26, 1938.

[13] K.-K. K. Kim, D. E. Shen, Z. K. Nagy and R. D. Braatz, "Wiener's Polynomial Chaos for the Analysis and Control of Nonlinear Dynamical Systems with Probabilistic Uncertainties [Historical Perspectives]," IEEE Control Systems Magazine, vol. 33, pp. 58-67, 2013.

[14] S. Oladyshkin and W. Nowak, "Data-driven Uncertainty Quantification Using the Arbitrary Polynomial Chaos Expansion," Reliability Engineering and System Safety, vol. 106, pp. 179190, 2012.

[15] F. Augustin, A. Gilg, M. Paffrath, P. Rentrop and U. Wever, "Polynomial Chaos for the Approximation of Uncertainties: Chances and Limits." European Journal of Applied Mathematics, vol. 19, pp. 149-190, 2008.

[16] K. K. Kim and R. D. Braatz, "Generalized Polynomial Chaos Expansion Approaches to Approximate Stochastic Receding Horizontal Control with Applications to Probabilistic Collision Checking and Avoidance.," in Proceeding of the IEEE Conference on Control Applications, Dubrovnik,Croatia, 2012.

[17] R. G. Ghanem and P. D. Spanos, "Spectral Stochastic Finite-element Formulation for Reliability Analysis," Journal of Engineering Mechanics, vol. 117, pp. 23512372, 1991.

[18] Z. Nagy and R. Braatz, "Distributional Uncertainty Analysis Using Polynomial Chaos Expansions," in Proceedings of the IEEE Int. Symposium on Computer-Aided Control System Design, Japan, 2010.

[19] W. Ray, Advanced Process Control, New York: McGraw-Hill, 1981.

(C)Bangladesh Uni. of Engg. \& Tech. 